Research Paper

\title{
Clustering Patterns of Comorbidities Associated with In-Hospital Death in Hospitalizations of US Adults with Venous Thromboembolism
}

James Tsai ${ }^{\bowtie}$, Althea M. Grant ${ }^{1}$, J. Michael Soucie ${ }^{1}$, Amy Helwig ${ }^{2}$, Hussain R. Yusuf ${ }^{1}$, Sheree L. Boulet ${ }^{3}$, Nimia L. Reyes ${ }^{1}$, Hani K. Atrash ${ }^{1}$

1. Division of Blood Disorders, National Center on Birth Defects and Developmental Disabilities Centers for Disease Control and Prevention, Atlanta, Georgia, USA;

2. Center for Quality Improvement and Patient Safety, Agency for Healthcare Research and Quality, Rockville, Maryland, USA;

3. Division of Reproductive Health, National Center for Chronic Disease Prevention and Health Promotion Centers for Disease Control and Prevention, Atlanta, Georgia, USA.

$\square$ Corresponding author: Dr. James Tsai, Division of Blood Disorders, National Center on Birth Defects and Developmental Disabilities Centers for Disease Control and Prevention, 1600 Clifton Road, Mail Stop E-64, Atlanta, GA 30333, USA. Fax:+1 404-498-6799; E-mail address: jxt9@cdc.gov

( ) Ivyspring International Publisher. This is an open-access article distributed under the terms of the Creative Commons License (http://creativecommons.org/ licenses/by-nc-nd/3.0/). Reproduction is permitted for personal, noncommercial use, provided that the article is in whole, unmodified, and properly cited.

Received: 2013.05.17; Accepted: 2013.07.29; Published: 2013.08.19

\begin{abstract}
Background: Venous thromboembolism (VTE) is a significant source of mortality, morbidity, disability, and impaired health-related quality of life in the world.

Objective: We aimed to evaluate the clustering patterns and associations of 29 comorbidities with in-hospital death among adult hospitalizations with a diagnosis of VTE in the United States by analyzing data from the 2009 Nationwide Inpatient Sample.

Methods: This cross-sectional study included 153,124 adult hospitalizations with a diagnosis of VTE. Adjusted rate ratios and $95 \%$ confidence intervals $(\mathrm{Cl})$ for in-hospital death were generated by using multivariable log-linear regression models to measure independent associations between comorbidities and in-hospital death.

Results: We estimated that 44,200 in-hospital deaths occurred in 2009 among 773,273 US adult hospitalizations with a diagnosis of VTE. Subgroups of hospitalizations with comorbidities of "congestive heart failure," "chronic pulmonary disease," "coagulopathy," "liver disease," "lymphoma," "fluid and electrolyte disorders," "metastatic cancer," "peripheral vascular disorders," "pulmonary circulation disorders," "renal failure," "solid tumor without metastasis," or "weight loss" were positively and independently associated with I.07 (95\% Cl: I.02-I.I2) to 2.06 (95\% Cl: 1.97-2.16) times increased likelihoods of in-hospital death, when compared to those without the corresponding comorbidities. The clustering patterns of these comorbidities by 4 disease categories (i.e., "cancer," "cardiovascular/respiratory/blood," "gastrointestinal/urologic," and "nutritional/bodyweight") were associated with 2.74 to 10.28 times increased likelihoods of in-hospital death, as compared to hospitalizations without any of these comorbidities. The overall increase in the cumulative number of comorbidities corresponded to significantly elevated risks $(P$-trend $<0.01)$ for in-hospital death among hospitalizations with a diagnosis of VTE.

Conclusion: The presence of multiple comorbidities is ubiquitous among hospitalizations of adults with VTE and among in-hospital deaths with VTE in the United States. The findings of our study further suggest that, among hospitalizations of adults with VTE, the presence of certain comorbidities or clustering of these comorbidities significantly elevates the risk of in-hospital death.
\end{abstract}

Key words: Comorbidity; Clustering pattern; Elixhauser comorbidity index; Venous thromboembolism; Hospitalization; Death; Mortality. 


\section{Introduction}

Venous thromboembolism (VTE), presenting clinically as pulmonary embolism or deep vein thrombosis, and its long-term sequelae of post-thrombotic syndrome and chronic thromboembolic pulmonary hypertension are significant sources of mortality, morbidity, disability, and impaired health-related quality of life in the world [1-4]. From the classic Virchow's triad (i.e., vascular endothelial damage, stasis of blood flow, and hypercoagulability of blood) to the current classification scheme of unprovoked (e.g., idiopathic) and provoked (e.g., secondary) VTE resulting from transient (e.g., fractures) or persistent risk factors (e.g., cancer) [5-7], research over the past several decades has unveiled that VTE is a multifaceted disease affecting individuals across various socio-demographic groups $[4,7,8]$.

A previous study reported that, among patients with a confirmed episode of VTE, about $74 \%$ developed VTE in the outpatient setting and $37 \%$ of such outpatients had a recent hospitalization in the preceding 3 months [9]. Recent data from the National Hospital Discharge Survey linked VTE to more than half a million US adult hospitalizations annually during 2007-2009 [10]. The risk of VTE and its prognostic health consequences increase with age and are associated with comorbidity - the presence of additional disease in relation to an index disease such as VTE [4, 11-14]. Advances in healthcare research demonstrate that comorbidities can affect one or more diseases through the etiological mechanisms of direct causation (e.g., disease A is directly responsible for disease B), associated risk factors (e.g., correlated risk factors make concurrent diseases more likely to occur), heterogeneity (e.g., independent risk factors for either disease), and independence (e.g., some diagnostic features of the co-occurring diseases actually correspond to a third distinct disease) [11]. An increasing body of research shows that comorbidities may confound clinical assessment [15, 16], hinder timely diagnosis [17], and aggravate complications and recurrence [18-21], thereby placing patients with VTE at heightened risks for many debilitating health consequences including death [19].

To date, numerous studies have identified a myriad of biological, behavioral, health, and environmental risk factors that are independently associated with risk of developing VTE [4-8]. Yet, limited research has been conducted to evaluate the joint impact of VTE and various medical illnesses on mortality in hospitalized patients with VTE. To the best of our knowledge, no study has evaluated the pattern and concurrent impacts of comorbidities on in-hospital death among US adult hospitalizations with VTE. Therefore, this study aimed to evaluate the clustering patterns and associations of comorbidities with in-hospital death among adult hospitalizations with a diagnosis of VTE in the United States by analyzing data from the 2009 Nationwide Inpatient Sample (NIS).

\section{Materials and methods}

\section{Data Source}

The NIS is part of the Healthcare Cost and Utilization Project sponsored by the Agency for Healthcare Research and Quality (AHRQ). It is the largest all-payer inpatient care database and includes 5-8 million annual unweighted hospitalizations of patients covered by Medicare, Medicaid, private insurance, and the uninsured from about 1,000 community hospitals in the United States. The NIS is designed to approximate a $20 \%$ sample of US community hospitals; all hospitalizations from sampled hospitals are included in the NIS [22]. The NIS sampling frame consists of non-federal, short-term, general, specialty hospitals, and long-term acute care facilities. Excluded from the NIS are short-term rehabilitation hospitals, long-term non-acute care hospitals, psychiatric hospitals, and alcoholism/chemical dependency treatment facilities [22]. Details about the sampling methodology are described elsewhere [23]. All sampling procedures involving human subjects and confidentiality were reviewed and approved by the Research Ethics Review Board of the AHRQ [23]. The 2009 Inpatient Core file contains data for 7,810,762 hospitalizations drawn from 1,050 hospitals of participating states that comprise $96 \%$ of the US population.

\section{Inpatient Sample}

Each sampled hospitalization in the NIS includes a maximum of 25 diagnostic codes based on the International Classification of Diseases, Ninth Revision, Clinical Modification (ICD-9-CM). VTE was identified using ICD-9-CM codes 415.1x, 451.1x, 451.2, 451.8x, $451.9,453.2,453.4 x, 453.8 x$, and 453.9 in any of the diagnostic fields. We limited our analysis to an unweighted sample of hospitalizations for adults aged 18 years and older with a diagnosis of VTE $(n=154,047)$. We excluded hospitalizations related to pregnancy, childbirth, and puerperium of patients who are mostly young, who have a low incidence of comorbidities and VTE, and who have a small risk of death during hospitalization, although the risk of developing VTE may be increased during pregnancy and postpartum period [24-27]. After excluding hospitalizations due to pregnancy, childbirth, and puerperium and variables with missing information for 
sex, vital status, length of hospital stay, or status of primary expected payer, 153,124 unweighted hospitalizations were included in the analyses.

\section{Demographic, Clinical, and Insurance Char- acteristics}

The demographic variables included age, sex, and race/ethnicity (15\% missing data). Because of potential misclassification and some participating states do not report race/ethnicity information, national estimates for race/ethnicity should be interpreted with caution. In addition, we included clinical characteristics and insurance status (i.e., days of hospital stay, insurance status of primary expected payer, and status of operating room procedure) as covariates, because of their relevance to the outcome and prevention of VTE. The NIS assigns all ICD-9-CM procedure codes to 4 broad categories of minor diagnostic, minor therapeutic, major diagnostic, and major therapeutic according to the AHRQ Procedure Classes [28]. An operating room procedure is defined in the NIS as having at least one major diagnostic or major therapeutic procedure during hospitalization [29].

\section{Comorbidity}

Comorbidities are considered in the NIS as coexisting medical conditions that are not directly related to the principal diagnosis or the main reason for admission, and are likely to have originated prior to the hospital stay [30]. Charlson et al and Elixhauser et al developed two well-known comorbidity indices for risk adjustment in health outcomes research [24, 25]. Lately, the Elixhauser comorbidity index has been found superior for risk adjustment and has been increasingly used in administrative datasets [31-34]. While the original Elixhauser comorbidity index contained 30 comorbidities based on ICD-9-CM coding algorithms for diagnoses and diagnosis-related group, the NIS included 29-comorbidity variables excluding "cardiac arrhythmias" [25, 30]. We constructed an ordinal variable to indicate the total number of AHRQ comorbidities for each hospitalization and categorized the variable into groups with 1, 2, 3, 4, 5 or more comorbidities.

\section{Statistical Analysis}

We calculated the percentage distribution and case-fatality rates of adult hospitalizations with a diagnosis of VTE stratified by age, sex, race/ethnicity, days of hospital stay, insurance status of primary expected payer, operating room procedure, and number of comorbidities. Regardless of actual causes of death, we defined the case-fatality rate in this study as the percentage of hospitalizations of patients with a VTE diagnosis who died during the study period [35]. We estimated the number of deaths with a diagnosis of VTE overall in 2009 and by number of comorbidities. We calculated the prevalence of each of the 29 comorbidities among adult hospitalizations. To identify comorbidities that were independently associated with in-hospital death in the study sample, we employed a backward elimination procedure to remove any explanatory variable with the highest $P \geq 0.05$ for individual $t$-test of null hypothesis $\beta=0$. The same procedure was repeated until $P<0.05$ for all explanatory variables remaining in the final model. Adjusted rate ratios (aRRs) and 95\% confidence intervals (CI) for in-hospital death were generated by using multivariate log-linear regression models to measure independent associations between remaining comorbidities and in-hospital death while controlling for age, sex, race/ethnicity, days of hospital stay, primary expected payer, and status of operating room procedure. We calculated the rate ratios for in-hospital death by number of comorbidities and by cumulative severity scores among adult hospitalizations with VTE. We assessed the distribution of 16 clustering patterns for 12 comorbidities by 4 disease categories (i.e., "cancer," "cardiovascular/respiratory/blood," "gastrointestinal/urologic," and "nutritional/ bodyweight") and calculated adjusted rate ratios (aRRs) for in-hospital death among adult hospitalizations with a diagnosis of VTE. We performed orthogonal polynomial contrasts to test linear and quadratic trends to indicate the increase or decrease of aRRs across the categories of comorbidity while controlling for covariates. We performed the data management and analysis using SPSS 18 Complex Samples for Survey Analysis (IBM Corp) and STATA 11 (StataCorp LP) to account for multiple stages of sampling, weighting, stratification, and clustering. All estimates in this study were weighted unless otherwise noted as unweighted sample size (n).

\section{Results}

We estimated that 44,200 in-hospital deaths occurred in 2009 among adult hospitalizations with a diagnosis of VTE in the United States, representing an overall case-fatality rate of $5.7 \%$. Approximately $97.9 \%$ of these deaths had at least 1 comorbidity (Figure 1). The case-fatality rates varied significantly by age, sex, race/ethnicity, days of hospital stay, insurance status of primary expected payer, status of operating room procedure, and the number of comorbidities $(P<0.001)$ (Table 1). For instance, large percentages of hospitalizations with VTE were observed among subgroups for patients who were 50-79 years of age, female, white, less than 7 days of hospital stay, or with "no procedure of any kind." However, high case-fatality rates were observed among subgroups 
with 80 years and older, male, Asian or other race/ethnicity, or "non-operating room procedure." Additionally, the case-fatality rates were higher among subgroups of patients whose primary expected payer was Medicare or who had at least 5 comorbidities than among those with other insurance payers or with fewer number of comorbidities $(P<$ 0.001) (Table 1).

Of 29 comorbidities from the AHRQ comorbidity index, 12 comorbidities were significantly and positively associated with in-hospital death among hospitalizations with VTE, after adjustment of covariates including age, sex, race/ethnicity, days of hospital stay, primary expected payer, status of operating room procedure, and remaining comorbidities (Table 2). For example, when compared to subgroups without corresponding comorbidity, subgroups with comorbidity of "congestive heart failure," "chronic pulmonary disease," "coagulopathy," "liver disease," "lymphoma," "fluid and electrolyte disorders," "metastatic cancer," "peripheral vascular disorders," "pulmonary circulation disorders," "renal failure," "solid tumor without metastasis," and "weight loss" were each independently associated with 1.07 to 2.06 times increased likelihoods of in-hospital death. The prevalence for having any one of 12 comorbidities ranged from $1.6 \%$ for "lymphoma" to $28.3 \%$ for "fluid and electrolyte disorders," whereas the case-fatality rates fluctuated between $6.8 \%$ for "chronic pulmonary disease" and $13.6 \%$ for "coagulopathy" (Table 2). Furthermore, the increase in cumulative number of comorbidities generally corresponded to significantly elevated risks of in-hospital death among hospitalizations with VTE $(P$-trend $<0.01)$ (Figure 2$)$. The detection of a significant quadratic trend with an accompanying linear trend $(P<0.001)$ signaled the presence of nonlinear variation (e.g., trend flattened out or leveled off) along with an overall linear trend (Figure 2). The disease category of "cardiovascular/blood/ respiratory," either by itself $(21.8 \%)$ or combined with the disease categories for other comorbidities (27.6\%) (i.e., "cancer," "gastrointestinal/renal," and "nutritional/body weight"), was most prevalent and accounted for nearly half $(49.4 \%)$ of adult hospitalizations with VTE. The clustering patterns of 12 comorbidities by 4 disease categories were associated with 2.74 to 10.28 times increased likelihoods of in-hospital death, as compared to adult hospitalizations without any of the 12 comorbidities (Table 3). Regardless, $28.9 \%$ adult hospitalizations with VTE did not have any of the 12 comorbidities of concern.

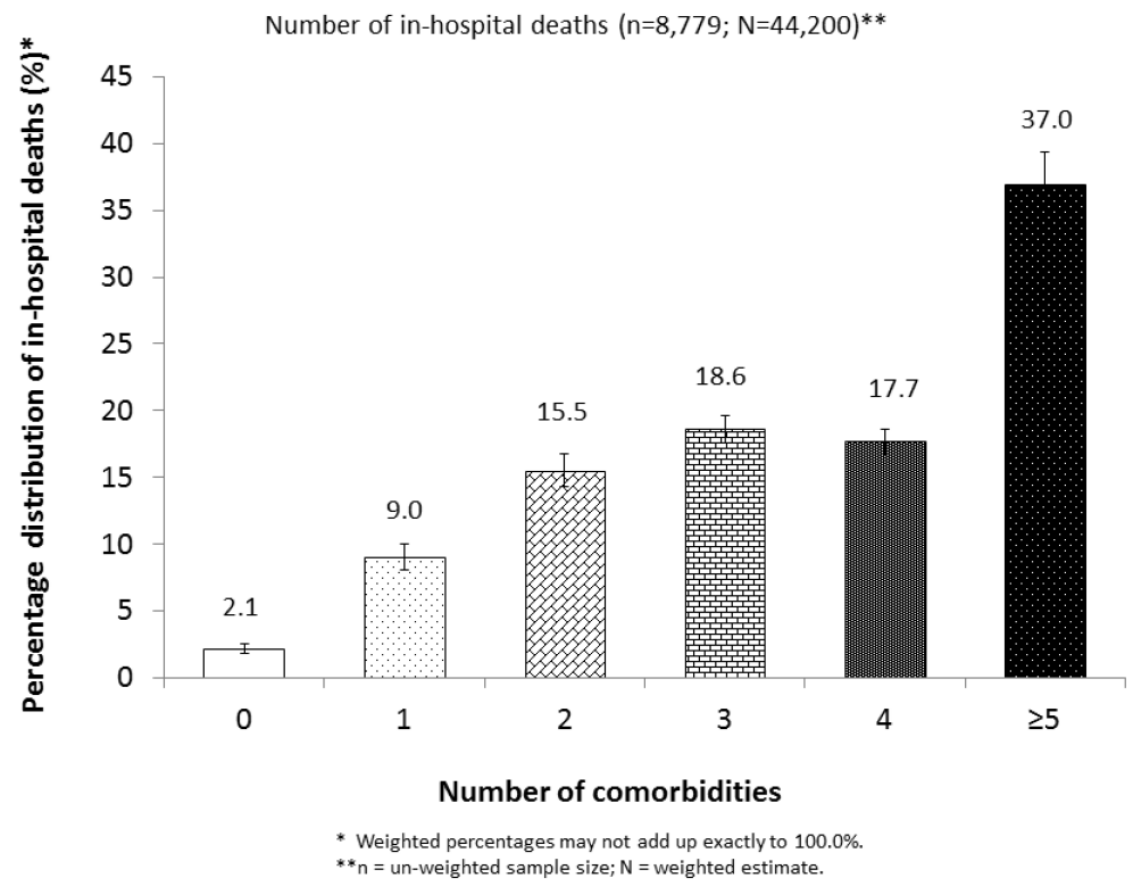

Figure I. Percentage distribution of estimated number of in-hospital deaths with VTE by status of comorbidity, NIS, 2009 


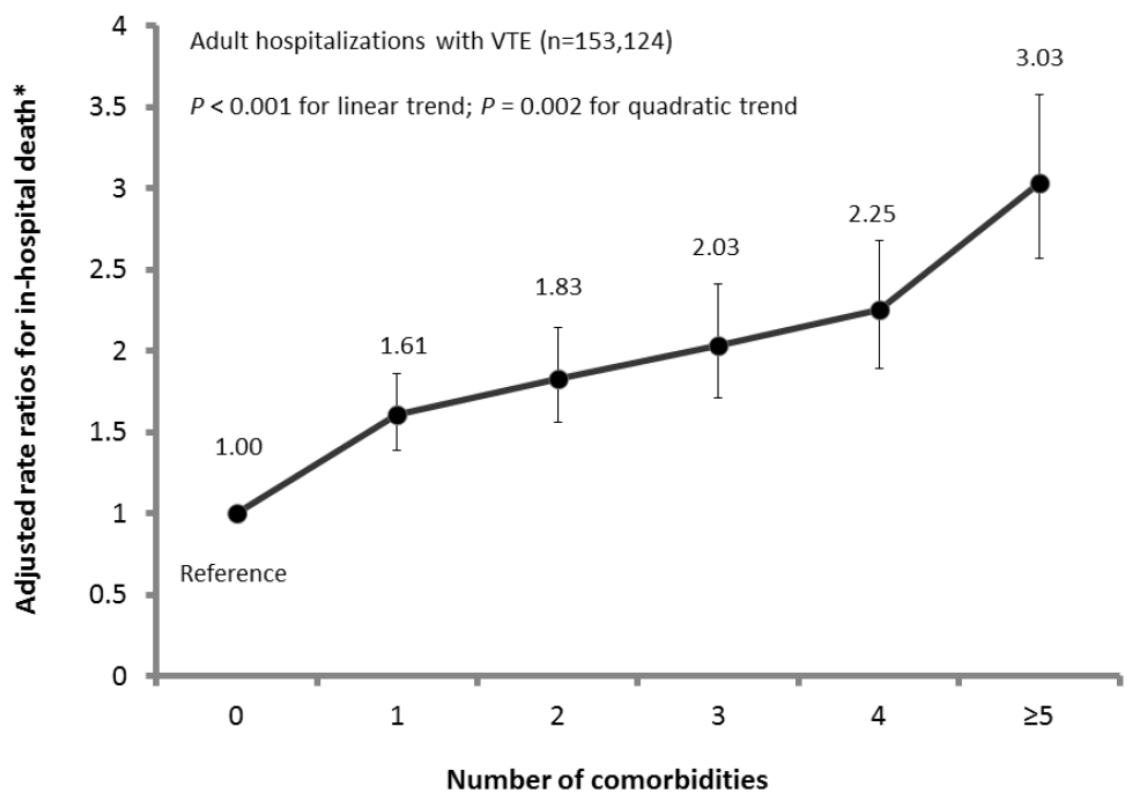

*Adjusted for age, sex, race/ethnicity, days of hospital stay, primary expected payer, and operating room procedure.

Figure 2. Adjusted rate ratios for in-hospital death by number of comorbidities among US adult hospitalizations with VTE, NIS, 2009

Table I. Percentage distribution and case-fatality rates of adult hospitalizations with a diagnosis of VTE by demographic and clinical characteristics, NIS, 2009

\begin{tabular}{|c|c|c|c|c|c|c|}
\hline \multirow[t]{2}{*}{ Characteristics } & \multirow{2}{*}{$\begin{array}{l}\text { Unweighted } \\
\mathbf{n}\end{array}$} & \multicolumn{2}{|c|}{ Estimated distribution } & \multicolumn{3}{|c|}{ In-hospital death $\left(\mathrm{N}^{\mathrm{a}}=44,200\right)$} \\
\hline & & $\mathbf{N}$ & $\%$ & Case-fatality (\%) & $\mathrm{CI}^{\mathrm{b}}$ & $P$-value \\
\hline Overall & 153,124 & 773,273 & 100 & 5.7 & $5.5-5.9$ & \\
\hline Age & & & & & & $P<0.001$ \\
\hline $18-49$ & 32,448 & 163,007 & 21.1 & 2.7 & $2.5-3.0$ & \\
\hline $50-79$ & 87,401 & 441,227 & 57.1 & 6.0 & $5.7-6.2$ & \\
\hline$\geq 80$ & 33,275 & 169,039 & 21.9 & 7.9 & $7.5-8.3$ & \\
\hline Sex & & & & & & $P<0.001$ \\
\hline Male & 73,324 & 369,954 & 47.9 & 6.0 & $5.8-6.3$ & \\
\hline Female & 79,800 & 403,319 & 52.1 & 5.4 & $5.2-5.7$ & \\
\hline Race/ethnicity ${ }^{\mathrm{d}}$ & & & & & & $P<0.001$ \\
\hline White & 93,654 & 473,796 & 61.3 & 5.6 & $5.4-5.9$ & \\
\hline Black & 20,814 & 105,294 & 13.6 & 5.8 & $5.4-6.2$ & \\
\hline Hispanic & 9,074 & 45,842 & 5.9 & 6.1 & $5.6-6.8$ & \\
\hline Asian or other race/ethnicity & 6,289 & 32,321 & 4.2 & 7.8 & $7.0-8.6$ & \\
\hline Not stated & 23,293 & 116,020 & 15.0 & 5.3 & $4.7-6.0$ & \\
\hline Days of hospital stay & & & & & & $P<0.001$ \\
\hline$<7$ days & 83,673 & 422,955 & 54.7 & 4.3 & $4.1-4.5$ & \\
\hline$\geq 7$ days & 69,451 & 350,318 & 45.3 & 7.5 & $7.2-7.8$ & \\
\hline Primary expected payer & & & & & & $P<0.001$ \\
\hline Medicare & 85,207 & 431,696 & 55.8 & 6.7 & $6.4-6.9$ & \\
\hline Medicaid & 13,829 & 69,660 & 9.0 & 4.8 & $4.4-5.2$ & \\
\hline Private including $\mathrm{HMO}$ & 42,151 & 212,229 & 27.4 & 4.5 & $4.2-4.8$ & \\
\hline Self-pay or other payers & 11,937 & 59,688 & 7.7 & 4.4 & $4.0-4.9$ & \\
\hline Operating room procedure & & & & & & $P<0.001$ \\
\hline No procedure of any kind recorded & 61,111 & 309,521 & 40.0 & 1.9 & $1.8-2.1$ & \\
\hline $\begin{array}{l}\text { Non-operating room procedure } \\
\text { recordede }\end{array}$ & 48,877 & 246,454 & 31.9 & 9.5 & $9.1-9.9$ & \\
\hline Operating room procedure recorded ${ }^{f}$ & 43,136 & 217,298 & 28.1 & 6.6 & $6.3-6.9$ & \\
\hline Number of comorbiditiesg & & & & & & $P<0.001$ \\
\hline 0 & 10,998 & 55,743 & 7.2 & 1.7 & $1.4-2.0$ & \\
\hline 1 & 23,177 & 117,449 & 15.2 & 3.4 & $3.1-3.7$ & \\
\hline 2 & 30,808 & 156,034 & 20.2 & 4.4 & $4.1-4.8$ & \\
\hline 3 & 30,831 & 154,254 & 19.9 & 5.3 & $5.5-5.7$ & \\
\hline 4 & 24,205 & 122,067 & 15.8 & 6.4 & $6.0-6.8$ & \\
\hline$\geq 5$ & 33,405 & 167,726 & 21.7 & 9.7 & $9.3-10.2$ & \\
\hline
\end{tabular}


Table 2. Prevalence of comorbidity, case-fatality rates and rate ratios for in-hospital death among adult hospitalizations with a diagnosis of VTE, NIS, 2009

\begin{tabular}{|c|c|c|c|c|c|c|c|c|}
\hline \multirow[t]{2}{*}{ AHRQ comorbidity index ${ }^{a}$} & \multirow[t]{2}{*}{$\begin{array}{l}\text { Unweighted } \\
n=153,124^{b}\end{array}$} & \multirow[t]{2}{*}{$\begin{array}{l}\text { Estimated } \\
\mathbf{N}\end{array}$} & \multicolumn{2}{|c|}{$\begin{array}{l}\text { Prevalence of comor- } \\
\text { bidity }\end{array}$} & \multicolumn{4}{|c|}{ Association with in-hospital death } \\
\hline & & & $\%$ & $95 \% \mathrm{CI} c$ & Case-fatality rate & $95 \% \mathrm{CI}$ & Adjusted RRd & $95 \% \mathrm{CI}$ \\
\hline Acquired immune deficiency syndrome & 549 & 2,756 & 0.4 & $0.3-0.4$ & 3.7 & $2.3-5.9$ & $-e$ & - \\
\hline Alcohol abuse & 4,868 & 24,442 & 3.2 & $3.0-3.3$ & 4.7 & $4.2-5.3$ & 0.84 & $0.74-0.95$ \\
\hline Anemia (deficiency) & 38,261 & 192,405 & 24.9 & $23.9-25.8$ & 5.9 & $5.6-6.3$ & 0.72 & $0.68-0.76$ \\
\hline $\begin{array}{l}\text { Arthritis (rheumatoid)/collagen vascular } \\
\text { diseases }\end{array}$ & 5,209 & 26,254 & 3.4 & $3.3-3.5$ & 4.9 & $4.4-5.6$ & - & - \\
\hline Chronic blood loss anemia & 2,904 & 14,636 & 1.9 & $1.8-2.0$ & 5.6 & $4.8-6.6$ & 0.66 & $0.57-0.77$ \\
\hline Congestive heart failure $f$ & 19,627 & 99,176 & 12.8 & $12.4-13.3$ & 10.8 & $10.3-11.4$ & 1.43 & $1.36-1.50$ \\
\hline Chronic pulmonary disease & 32,301 & 163,376 & 21.1 & $20.5-21.7$ & 6.8 & $6.4-7.2$ & 1.07 & $1.02-1.12$ \\
\hline Coagulopathy & 12,685 & 63,524 & 8.2 & $7.8-8.6$ & 13.6 & $12.8-14.4$ & 1.84 & $1.74-1.94$ \\
\hline Depression & 15,878 & 80,027 & 10.3 & $10.0-10.7$ & 3.6 & $3.3-4.0$ & 0.72 & $0.67-0.79$ \\
\hline Diabetes, uncomplicated & 29,750 & 150,104 & 19.4 & $19.0-19.9$ & 5.6 & $5.3-6.0$ & - & - \\
\hline Diabetes with chronic complications & 6,936 & 34,879 & 4.5 & $4.3-4.8$ & 5.5 & $4.9-6.1$ & 0.83 & $0.75-0.92$ \\
\hline Drug abuse & 3,806 & 19,116 & 2.5 & $2.3-2.7$ & 3.3 & $2.8-4.0$ & 0.80 & $0.67-0.95$ \\
\hline $\begin{array}{l}\text { Hypertension (uncomplicated and com- } \\
\text { plicated) }\end{array}$ & 82,484 & 416,519 & 53.9 & $53.0-54.7$ & 5.0 & $4.7-5.2$ & 0.70 & $0.66-0.73$ \\
\hline Hypothyroidism & 16,938 & 85,473 & 11.1 & $10.7-11.4$ & 5.0 & $4.6-5.4$ & 0.88 & $0.82-0.94$ \\
\hline Liver disease & 3,722 & 18,665 & 2.4 & $2.3-2.6$ & 7.9 & $7.0-8.9$ & 1.17 & $1.04-1.32$ \\
\hline Lymphoma & 2,512 & 12,719 & 1.6 & $1.5-1.8$ & 9.4 & $8.3-10.7$ & 1.43 & $1.27-1.62$ \\
\hline Fluid and electrolyte disorders & 43,505 & 218,526 & 28.3 & $27.4-29.1$ & 10.8 & $10.4-11.2$ & 1.90 & $1.82-1.98$ \\
\hline Metastatic cancer & 10,949 & 55,362 & 7.2 & $6.9-7.5$ & 11.4 & $10.7-12.1$ & 1.89 & $1.77-2.01$ \\
\hline Other neurological disorders & 14,270 & 72,079 & 9.3 & $9.1-9.6$ & 6.2 & $5.8-6.7$ & - & - \\
\hline Obesity & 18,744 & 94,178 & 12.2 & $11.7-12.7$ & 3.8 & $3.5-4.1$ & 0.86 & $0.79-0.93$ \\
\hline Paralysis & 6,356 & 31,978 & 4.1 & $3.9-4.4$ & 6.9 & $6.2-7.6$ & 1.10 & $1.00-1.21$ \\
\hline Peripheral vascular disorders & 9,322 & 46,873 & 6.1 & $5.8-6.3$ & 8.3 & $7.7-8.9$ & 1.21 & $1.13-1.30$ \\
\hline Psychoses & 6,390 & 32,150 & 4.2 & $4.0-4.4$ & 4.1 & $3.6-4.7$ & 0.79 & $0.70-0.90$ \\
\hline Pulmonary circulation disorders & 30,267 & 152,273 & 19.7 & $19.2-20.2$ & 11.0 & $10.5-11.5$ & 2.06 & $1.97-2.16$ \\
\hline Renal failure & 23,036 & 116,090 & 15.0 & $14.5-15.6$ & 8.2 & $7.8-8.7$ & 1.38 & $1.30-1.45$ \\
\hline Solid tumor without metastasis & 7,487 & 37,893 & 4.9 & $4.7-5.1$ & 7.6 & $6.9-8.2$ & 1.34 & $1.23-1.47$ \\
\hline Peptic ulcer disease excluding bleeding & 73 & 371 & 0 & $0-0.1$ & - & - & - & - \\
\hline Valvular disease & 6,804 & 34,318 & 4.4 & $4.2-4.7$ & 7.5 & $6.8-8.3$ & 0.86 & $0.79-0.94$ \\
\hline Weight loss & 14,080 & 70,584 & 9.1 & $8.6-9.7$ & 13.1 & $12.4-13.8$ & 1.39 & $1.32-1.47$ \\
\hline
\end{tabular}

Table 3. Distribution of clustering patterns for 12 comorbidities by disease category and rate ratios for in-hospital death among adult hospitalizations with a diagnosis of VTE, NIS, 2009

\begin{tabular}{|c|c|c|c|c|c|c|c|c|c|}
\hline \multirow[t]{2}{*}{$\begin{array}{l}\text { Total \# of catego- } \\
\text { ries }\end{array}$} & Cancera & $\begin{array}{l}\text { Cardiovascular } \\
\text { /Blood/Respiratoryb }\end{array}$ & $\begin{array}{l}\text { Gastrointestinal } \\
\text { /Renalc }\end{array}$ & $\begin{array}{l}\text { Nutritional } \\
\text { /Bodyweightd }^{\mathrm{d}}\end{array}$ & $\begin{array}{l}\text { Unweighted } \\
\mathrm{n}\end{array}$ & \multicolumn{2}{|c|}{$\begin{array}{l}\text { Estimated distribu- } \\
\text { tion }\end{array}$} & \multicolumn{2}{|c|}{ In-hospital death } \\
\hline & \multicolumn{3}{|c|}{ Clustering pattern } & & & $\mathrm{N}$ & $\%$ & aRRe & $\mathrm{CI}$ \\
\hline 4 & $+\mathrm{f}$ & + & + & + & 740 & 3,730 & 0.5 & 10.28 & $8.66-12.20$ \\
\hline 3 & + & + & + & $-g$ & 667 & 3,380 & 0.4 & 6.38 & $4.97-8.19$ \\
\hline 3 & - & + & + & + & 6,441 & 32,299 & 4.2 & 7.85 & $7.00-8.79$ \\
\hline 3 & + & - & + & + & 453 & 2,282 & 0.3 & 6.86 & $5.40-8.72$ \\
\hline 3 & + & + & - & + & 3,795 & 19,138 & 2.5 & 8.45 & $7.51-9.50$ \\
\hline 2 & + & + & - & - & 5,201 & 26,313 & 3.4 & 5.00 & $4.42-5.67$ \\
\hline 2 & - & + & + & - & 7,344 & 37,045 & 4.8 & 3.73 & $3.29-4.24$ \\
\hline 2 & - & - & + & + & 3,707 & 18,677 & 2.4 & 4.41 & $3.81-5.11$ \\
\hline 2 & + & - & + & - & 652 & 3,302 & 0.4 & 3.12 & $2.23-4.38$ \\
\hline 2 & + & - & - & + & 3,091 & 15,626 & 2.0 & 5.44 & $4.71-6.27$ \\
\hline 2 & - & + & - & + & 17,815 & 89,284 & 11.5 & 6.10 & $5.48-6.80$ \\
\hline 1 & + & - & - & - & 6,203 & 31,452 & 4.1 & 2.46 & $2.11-2.86$ \\
\hline 1 & - & + & - & - & 33,267 & 168,271 & 21.8 & 2.74 & $2.48-3.03$ \\
\hline 1 & - & - & + & - & 6,032 & 30,422 & 3.9 & 1.65 & $1.35-2.01$ \\
\hline 1 & - & - & - & + & 13,715 & 68,954 & 8.9 & 2.98 & $2.66-3.34$ \\
\hline 0 & - & - & - & - & 44,001 & 223,098 & 28.9 & 1.00 & Reference ${ }^{h}$ \\
\hline \multicolumn{5}{|c|}{ Total number of hospitalizations } & 153,124 & 773,273 & 100.0 & - & - \\
\hline
\end{tabular}


a. Presence of one or more comorbidities of lymphoma, metastatic cancer, and solid tumor without metastasis. b. Presence of one or more comorbidities of congestive heart failure, chronic pulmonary disease, coagulopathy, peripheral vascular disorders, and pulmonary circulation disorders. c. Presence of one or more comorbidities of liver disease and renal failure. $\mathrm{d}$. Presence of one or more comorbidities of fluid and electrolyte disorders and weight loss. e. Rate ratios from log-linear regression model that adjusted for age, sex, race/ethnicity, days of hospital stay, primary expected payer, major operating room procedure, and comorbidities retained without 12 comorbidities. f. Presence of one or more comorbidities of the disease category. g. Absence of any comorbidity of the disease category. h. Absence of 12 comorbidities.

\section{Discussion}

By using a large nationally representative sample, our study evaluated the AHRQ 29-comorbidity index encompassing the clustering patterns and associations with in-hospital death among US adult hospitalizations with a diagnosis of VTE that have not been reported in the past. Of US adult hospitalizations with VTE, about 9 in 10 had at least 1 comorbidity and 1 in 3 had at least 4 comorbidities. A vast majority of in-hospital deaths with VTE had at least 1 comorbidity. From the well-recognized "metastatic cancer (aRR=1.89; CI: $1.77-2.01)^{\prime \prime}$ to the less pronounced "fluid and electrolyte disorders (aRR=1.90; CI: 1.82-1.98)," we identified 12 of these 29 comorbidities that were significantly associated with the risk of in-hospital deaths. In addition, increases in the cumulative number of comorbidities were associated with significantly elevated risks of in-hospital death among those hospitalizations.

The findings of our study have several important implications for clinical and public health research, practice, and planning. First, the ubiquitous presence of co-morbidities among patients with VTE suggests that, to a great extent, VTE is an important marker or reminder for the co-existence of other diseases or conditions. For instance, VTE may reflect the presence of a biologically more aggressive cancer in an early stage [14]. Therefore, healthcare practitioners must remain vigilant in evaluating patients with VTE, particularly those who may have clustering comorbidities that may predispose them to a heightened risk for death. Second, the breadth of co-morbidities among hospitalizations of patients with VTE demonstrates the complexity of potential disease-interacting mechanisms of (a) direct causation (e.g., cancer may compress adjacent veins to limit blood flow and produce procoagulant proteins and inflammatory cytokines that contribute to hypercoagulability) [36, 37], (b) associated risk factors (e.g., VTE and congestive heart failure may co-occur because inflammation and coronary heart disease are correlated risk factors, respectively) [38-40], (c) heterogeneity (e.g., aging and trauma are independent risk factors for either VTE or fluid and electrolyte disorders) [41, 42], and (d) independence (e.g., concurrent weight loss and renal failure resulting from cancer) [43]. As such, comorbidities not only have value in diagnostic and prognostic assessment of patients with VTE [11, 44, 45], but can also affect quality of patient care (e.g., adherence to clinical guidelines) [46, 47]. Third, we observed that almost half $(49.4 \%)$ of adult hospitalizations with VTE also had at least one comorbidity in the "cardiovascular/blood/respiratory" disease category. Research suggests that concordant comorbidities are those diseases that may share overlapping pathophysiologic risk factors, thus benefit from similar disease management strategies and may result in improved quality of patient care [48, 49]. For example, arterial thrombosis and VTE are two distinct forms of thrombosis with interrelated risk factors [50]. The JUPITER study reported that the use of statins resulted in a $43 \%$ reduction of VTE events in apparently healthy adults aged 50 years and older with high levels of C-reactive protein ( $\geq 2 \mathrm{mg} / \mathrm{L}$ ) and normal levels of low-density lipoprotein cholesterol [51]. In contrast, discordant comorbidities are not directly related in either pathogenesis or management of relevant diseases. Because of the additional implementation burden from multiple clinical recommendations or practice guidelines, competing demands for physician attention, and elevated risks of adverse drug events or complications, discordant comorbidities may lead to reduced quality of patient care $[48,49]$. Hence, substantial work remains for practitioners and researchers to elucidate the role of interacting VTE and comorbidities, to prioritize care of complex patients, and to inform prevention programs.

Based on the ascertainment of up to 25 diagnostic codes for each hospitalization in the NIS sample, a total of 44,200 in-hospital deaths were approximated among adult hospitalizations with VTE in 2009. Similar to the findings of our study, the 12 comorbidities overlapped with some comorbidities that were previously found to be associated with increased risks for in-hospital death in studies using a non-VTE-specific overall sample [31, 52]. Possibly as a surrogate for relatively "healthy" patients when compared to those with more serious diseases, the presence of the 11 non-life-threatening comorbidities in our study was associated with decreased risks of in-hospital death (Table 2). Although the reason remained unclear for the lower case-fatality rate in hospitalization with "operating room procedure" than that of "non-operating room procedure," additional research discerning these risk factors is needed in order to identify potential diagnostic and therapeutic procedures (e.g., venous catheterization) and associated conditions that may contribute to an elevated risk of death.

Our study has several limitations. We were unable to determine any causal inference because the 
cross-sectional study design cannot establish such a relationship. Due to the constraints of administrative datasets, we were unable to control for potential medications that may otherwise affect short-term mortality in patients with VTE. Even though ICD-9 codes provide a uniform identification of comorbidities in administrative datasets, the accuracy of diagnostic codes may vary by physician documentations, code assignments, usage patterns for diagnostic tests, changes of codes over time, and institutional reimbursement policies [53].

More than $66 \%$ of all healthcare costs or $96 \%$ of Medicare expenditures are for individuals with two or more chronic diseases and conditions [54, 55]. Also, given that comorbidities are highly prevalent among aging US adults and are associated with worse health outcomes and more complex clinical management, effectively addressing multiple chronic diseases and conditions poses a complex set of challenges for clinicians, patients, and healthcare systems [54-56]. More results from intervention studies that can demonstrate efficacy and safety in the presence of co-occurring diseases are beneficial.

\section{Conclusion}

The presence of multiple comorbidities is ubiquitous among hospitalizations of adults with VTE and among in-hospital deaths with VTE in the United States. The findings of our study further suggest that, among hospitalizations of adults with VTE, the presence of certain comorbidities or clustering of these comorbidities significantly elevates the risk of in-hospital death.

\section{Acknowledgements}

The authors of this study sincerely thank the coordinators of the Healthcare Cost and Utilization Project for all participating states, Agency for Healthcare Research and Quality, Rockville, MD, for making the data available.

\section{Authors' contributions}

All authors have fulfilled the criteria for authorship. JT, AMG, and JMS contributed to the study conception and design. JT had full access to all the data in the study and takes responsibility for the integrity of the data and the accuracy of the data analysis. JT performed data analyses, interpreted the results, and prepared the draft manuscript. JT, AMG, JMS, AH, HRY, SLB, NLR, and HKA made critical revisions of the manuscript for important intellectual content. AMG and HKA provided administrative, technical, material support, and study supervision. All authors have read and approved the final manuscript.

\section{Role of the Funding Source}

This study was conducted by employees of the Centers for Disease Control and Prevention and the Agency for Healthcare Research and Quality as part of their professional duties. No external funding was received for this study.

\section{Disclaimer}

The findings and conclusions in this paper are those of the authors and do not necessarily represent the official position of the Centers for Disease Control and Prevention or the Agency for Healthcare Research and Quality.

\section{Competing Interests}

The authors have declared that no competing interest exists.

\section{References}

1. [Internet] Office of the Surgeon General, US Department of Health \& Human Services. The Surgeon General's Call to Action to Prevent Deep Vein Thrombosis and Pulmonary Embolism. http://www.surgeongeneral.gov/topics/deepvein/calltoaction/call-to -action-on-dvt-2008.pdf.

2. Cohen AT, Tapson VF, Bergmann JF, Goldhaber SZ, Kakkar AK, Deslandes B, et al. Venous thromboembolism risk and prophylaxis in the acute hospital care setting (ENDORSE study): a multinational cross-sectional study. Lancet. 2008; 371: 387-94. doi:10.1016/S0140-6736(08)60202-0.

3. Tapson VF, Decousus H, Pini M, Chong BH, Froehlich JB, Monreal M, et al. Venous thromboembolism prophylaxis in acutely ill hospitalized medical patients: findings from the International Medical Prevention Registry on Venous Thromboembolism. Chest. 2007; 132: 936-45. doi:10.1378/chest.06-2993.

4. Heit JA. Venous thromboembolism: disease burden, outcomes and risk factors. J Thromb Haemost. 2005; 3: 1611-7. doi:JTH1415 [pii]10.1111/j.1538-7836.2005.01415.x.

5. White RH. Identifying risk factors for venous thromboembolism. Circulation. 2012; 125: 2051-3. doi:10.1161/CIRCULATIONAHA.112.102814.

6. Anderson FA, Jr., Spencer FA. Risk factors for venous

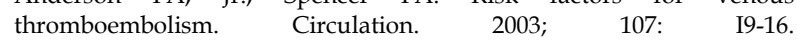
doi:10.1161/01.CIR.0000078469.07362.E6.

7. Goldhaber SZ. Risk factors for venous thromboembolism. J Am Coll $\begin{array}{llll}\text { Cardiol. 2010; 56: 1-7. doi:S0735-1097(10)01525-1 } & \end{array}$ [pii]10.1016/j.jacc.2010.01.057.

8. Rosendaal FR. Venous Thrombosis: The Role of Genes, Environment, and Behavior. Hematology. 2005; 2005: 1-12. doi:10.1182/asheducation-2005.1.1.

9. Spencer FA, Lessard D, Emery C, Reed G, Goldberg RJ. Venous thromboembolism in the outpatient setting. Arch Intern Med. 2007; 167: 1471-5. doi:10.1001/archinte.167.14.1471.

10. CDC. Venous Thromboembolism in Adult Hospitalizations - United States, 2007-2009. Centers for Disease Control and Prevention (CDC). MMWR Morb Mortal Wkly Rep. 2012; 61: 402-4.

11. Valderas JM, Starfield B, Sibbald B, Salisbury C, Roland M. Defining comorbidity: implications for understanding health and health services. Annals of family medicine. 2009; 7: 357-63. doi:10.1370/afm.983.

12. Tsai J, Grosse SD, Grant AM, Hooper WC, Atrash HK. Trends in in-hospital deaths among hospitalizations with pulmonary embolism. Arch Intern Med. 2012; 172: 960-1. doi:10.1001/archinternmed.2012.198.

13. Tsai J, Grosse SD, Grant AM, Reyes NL, Hooper WC, Atrash HK. Correlates of In-Hospital Deaths among Hospitalizations with Pulmonary Embolism: Findings from the 2001-2008 National Hospital Discharge Survey. PLOS ONE. 2012; 7: e34048. doi:10.1371/journal.pone.0034048.

14. Alcalay A, Wun T, Khatri V, Chew HK, Harvey D, Zhou H, et al. Venous thromboembolism in patients with colorectal cancer: incidence and effect on survival. J Clin Oncol. 2006; 24: 1112-8. doi:10.1200/JCO.2005.04.2150. 
15. Kelly J, Rudd A, Lewis RR, Hunt BJ. Plasma d-dimers in the diagnosis of venous thromboembolism. Arch Intern Med. 2002; 162: 747-56. doi:10-1001/pubs.Arch Intern Med.-ISSN-0003-9926-162-7-ira10030.

16. Kelly J, Hunt BJ, Rudd A, Lewis RR. Pulmonary embolism and pneumonia may be confounded after acute stroke and may co-exist. Age Ageing. 2002; 31: 235-9.

17. Smith SB, Geske JB, Morgenthaler TI. Risk Factors Associated with Delayed Diagnosis of Acute Pulmonary Embolism. The Journal of Emergency Medicine. 2012; 42: 1-6. doi:10.1016/j.jemermed.2011.06.004.

18. Palareti G, Cosmi B. Bleeding with anticoagulation therapy - who is at risk, and how best to identify such patients. Thromb Haemost. 2009; 102: 268-78. doi:10.1160/TH08-11-0730.

19. Gross CP, Galusha DH, Krumholz HM. The impact of venous thromboembolism on risk of death or hemorrhage in older cancer patients. J Gen Intern Med. 2007; 22: 321-6. doi:10.1007/s11606-006-0019-x.

20. Lin J, Proctor MC, Varma M, Greenfield LJ, Upchurch Jr GR, Henke PK. Factors associated with recurrent venous thromboembolism in patients with malignant disease. Journal of Vascular Surgery. 2003; 37: 976-83. doi:10.1067/mva.2003.191.

21. Nijkeuter $M$, Sohne $M$, Tick LW, Kamphuisen PW, Kramer MH, Laterveer $\mathrm{L}$, et al. The natural course of hemodynamically stable pulmonary embolism: Clinical outcome and risk factors in a large prospective cohort study. Chest. 2007; 131: 517-23. doi:10.1378/chest.05-2799.

22. [Internet] Agency for Healthcare Research and Quality (AHRQ). Introduction to the HCUP Nationwide Inpatient Sample (NIS); Healthcare Cost and Utilization Project (HCUP); 2009. http://www.hcup-us.ahrq.gov/db/nation/nis/NIS_Introduction_2009. jsp.

23. [Internet] Agency for Healthcare Research and Quality (AHRQ). Overview of the Nationwide Inpatient Sample (NIS). http://www.hcup-us.ahrq.gov/nisoverview.jsp.

24. Charlson ME, Pompei P, Ales KL, MacKenzie CR. A new method of classifying prognostic comorbidity in longitudinal studies: development and validation. Journal of chronic diseases. 1987; 40: 373-83.

25. Elixhauser A, Steiner C, Harris DR, Coffey RM. Comorbidity measures for use with administrative data. Med Care. 1998; 36: 8-27.

26. Krivak TC, Zorn KK. Venous thromboembolism in obstetrics and gynecology. Obstet Gynecol. 2007; 109: 761-77. doi:10.1097/01.AOG.0000255819.10187.70.

27. James A, Jamison M, Brancazio L, Myers E. Venous thromboembolism during pregnancy and the postpartum period: incidence, risk factors, and mortality. American journal of obstetrics and gynecology. 2006; 194 : 1311-5.

28. [Internet] Agency for Healthcare Research and Quality (AHRQ). Procedure Classes 2012. http://www.hcup-us.ahrq.gov/ toolssoftware/procedure/procedure.jsp.

29. [Internet] Agency for Healthcare Research and Quality (AHRQ). Major operating room procedure indicator. http://www.hcup-us.ahrq.gov/db/vars/orproc/nisnote.jsp.

30. [Internet] Agency for Healthcare Research and Quality (AHRQ). Comorbidity Software, Version 3.7. http://www.hcup-us.ahrq.gov/toolssoftware/comorbidity/comorbidit y.jsp.

31. Southern DA, Quan H, Ghali WA. Comparison of the Elixhauser and Charlson/Deyo methods of comorbidity measurement in administrative data. Med Care. 2004; 42: 355-60.

32. Dominick KL, Dudley TK, Coffman CJ, Bosworth HB. Comparison of three comorbidity measures for predicting health service use in patients with osteoarthritis. Arthritis and rheumatism. 2005; 53: 666-72. doi:10.1002/art.21440.

33. Chu YT, Ng YY, Wu SC. Comparison of different comorbidity measures for use with administrative data in predicting short- and long-term mortality. BMC health services research. 2010; 10: 140. doi:10.1186/1472-6963-10-140.

34. Tsai J, Abe K, Boulet SL, Beckman MG, Hooper WC, Grant AM. Predictive accuracy of 29-comorbidity index for in-hospital deaths in US adult hospitalizations with a diagnosis of venous thromboembolism. PLOS ONE. 2013; 8(7): e70061. doi:10.1371/journal.pone.0070061.

35. Rothman KJ, Greenland S, Lash T. Modern Epidemiology; Third Edition. Lippincott Williams \& Wilkins. 2008

36. Wun T, White RH. Venous thromboembolism (VTE) in patients with cancer: epidemiology and risk factors. Cancer investigation. 2009; 27 Suppl 1: 63-74. doi:10.1080/07357900802656681.

37. Kessler CM. The link between cancer and venous thromboembolism: a review. Am J Clin Oncol. 2009; 32: S3-7. doi:10.1097/COC.0b013e3181b01b17.
38. He J, Ogden LG, Bazzano LA, Vupputuri S, Loria C, Whelton PK. Risk factors for congestive heart failure in US men and women: NHANES I epidemiologic follow-up study. Arch Intern Med. 2001; 161: 996-1002.

39. Fox EA, Kahn SR. The relationship between inflammation and venous thrombosis. A systematic review of clinical studies. Thromb Haemost. 2005; 94: 362-5. doi:10.1267/THRO05020362.

40. Madjid $\mathrm{M}$, Willerson JT. Inflammatory markers in coronary heart disease. British Medical Bulletin. 2011; 100: 23-38. doi:10.1093/bmb/ldr043.

41. Luckey AE, Parsa CJ. Fluid and electrolytes in the aged. Archives of surgery. 2003; 138: 1055-60. doi:10.1001/archsurg.138.10.1055.

42. Lee JW. Fluid and electrolyte disturbances in critically ill patients. Electrolyte Blood Press. 2010; 8: 72-81. doi:10.5049/EBP.2010.8.2.72.

43. Patel P, Henry LL, Ganti AK, Potti A. Clinical course of lung cancer in patients with chronic kidney disease. Lung cancer. 2004; 43: 297-300. doi:10.1016/j.lungcan.2003.08.029.

44. Lugtenberg M, Burgers JS, Clancy C, Westert GP, Schneider EC. Current guidelines have limited applicability to patients with comorbid conditions: a systematic analysis of evidence-based guidelines. PLoS One. 2011; 6: e25987. doi:10.1371/journal.pone.0025987.

45. Boyd CM, Darer J, Boult C, Fried LP, Boult L, Wu AW. Clinical practice guidelines and quality of care for older patients with multiple comorbid diseases: implications for pay for performance. JAMA. 2005; 294: 716-24. doi:10.1001/jama.294.6.716

46. Tinetti ME, Bogardus ST, Jr., Agostini JV. Potential pitfalls of disease-specific guidelines for patients with multiple conditions. N Engl J Med. 2004; 351: 2870-4. doi:10.1056/NEJMsb042458.

47. McLaughlin TJ, Soumerai SB, Willison DJ, Gurwitz JH, Gao X, Borbas C, et al. The effect of comorbidity on use of thrombolysis or aspirin in patients with acute myocardial infarction eligible for treatment. Journal of general internal medicine. 1997; 12: 1-6.

48. Lagu T, Weiner MG, Hollenbeak CS, Eachus S, Roberts CS, Schwartz JS, et al. The impact of concordant and discordant conditions on the quality of care for hyperlipidemia. J Gen Intern Med. 2008; 23: 1208-13. doi:10.1007/s11606-008-0647-4

49. Min LC, Wenger NS, Fung C, Chang JT, Ganz DA, Higashi T, et al. Multimorbidity is associated with better quality of care among vulnerable elders. Med Care. 2007; 45: 480-8. doi:10.1097/MLR.0b013e318030fff9.

50. Ageno W, Becattini C, Brighton T, Selby R, Kamphuisen PW. Cardiovascular risk factors and venous thromboembolism: a $\begin{array}{llll}\text { meta-analysis. } & \text { Circulation. } & 2008 ; & 117 \text { : }\end{array}$ doi:10.1161/CIRCULATIONAHA.107.709204.

51. Glynn RJ, Danielson E, Fonseca FA, Genest J, Gotto AM, Jr., Kastelein JJ, et al. A randomized trial of rosuvastatin in the prevention of venous thromboembolism. N Engl J Med. 2009; 360: 1851-61. doi:10.1056/NEJMoa0900241.

52. van Walraven C, Austin PC, Jennings A, Quan H, Forster AJ. A modification of the Elixhauser comorbidity measures into a point system for hospital death using administrative data. Med Care. 2009; 47: 626-33. doi:10.1097/MLR.0b013e31819432e5.

53. Sarrazin MS, Rosenthal GE. Finding pure and simple truths with administrative data. JAMA. 2012; 307: 1433-5. doi:10.1001/jama.2012.404.

54. DHHS. Multiple Chronic Conditions: A Strategic Framework. Optimum Health and Quality of Life for Individuals with Multiple Chronic Conditions. Washington, DC: US Department of Health and Human Services (DHHS). 2010.

55. [Internet] Anderson G. Chronic Care: Making the Case for Ongoing Care. Princeton, NJ: Robert Wood Johnson Foundation. http://www.rwjf.org/files/research/50968chronic.care.chartbook.pdf.

56. Boyd CM, Fortin M. Future of Multimorbidity Research: How Should Understanding of Multimorbidity Inform Health System Design? Public Health Reviews. 2010; 32: 451-74. 